\title{
Complex Conductance of Carnauba Wax/Polyaniline Composites
}

\author{
J. Mariz G. Neto ${ }^{1}$, G. F. Leal Ferreira ${ }^{2}$, J. Ribeiro Santos Jr. ${ }^{3}$, \\ H. N. da Cunha ${ }^{1}$, I. F. Dantas ${ }^{1}$, and R. F. Bianchi ${ }^{2}$ \\ ${ }^{1}$ Departamento de Física, Universidade Federal do Piauí, \\ 64049-550 Teresina-PI, Brasil \\ ${ }^{2}$ Instituto de Física de São Carlos, \\ Universidade de São Paulo, 13560-970, São Carlos SP, Brasil \\ ${ }^{3}$ Departamento de Química, Universidade, Federal do Piauí, 64049-550 Teresina-PI, Brasil
}

Received on 24 July, 2002

\begin{abstract}
Complex conductance, $\sigma^{*}(f)=\sigma \prime(f)+i \sigma^{\prime \prime}(f)$, measurements of polyaniline/carnauba wax composites were carried out at room temperature in samples containing different amounts of PAni. While $\sigma$ " $(f)$ ever grows as a function of the frequency $f, \sigma \prime(f)$ was observed to vary as $f^{n}(n \approx 1)$ in the high frequency domain, and to be frequency-independent for lower frequencies. This is the quasi-universal behavior characteristic of conduction in a disordered medium. Superposed on this, another signal was observed to occur, and the whole impedance response was then analysed in an Argand Diagram, disclosing an electrode process in series with the bulk one. An equivalent conductance expression was used to explain such electrical behavior, in which the bulk properties of the composite were represented through the Random Free Energy Barrier model, while the composite-electrode interface by a simple parallel combination of a capacitor and a resistor. From the theoretical fittings it was concluded that the increase of PAni concentration acts in the sense of increasing not only the sample conductance, but also the bulk capacitance. The separation of the bulk and the interface contributions was then achieved.
\end{abstract}

\section{Introduction}

In recent years, many authors have pointed out the possibility of producing organic composites that associate the low cost and the mechanical properties of natural materials, as rubber or waxes, with the electronic properties of conducting polymers [1,2]. Among such materials, carnauba wax (cw) and polyaniline (PAni) appears as potential candidates to be used in this class of composites. While PAni presents high electric conductivity when doped by acid solutions [3,4], cw - a natural product of the carnauba palm (Copernicia cerifera) [5,6] - is a dielectric material produced in abundance in northeast of Brazil. In this context, the preparation of materials based on the mixture of PAni and cw appears as an alternative to obtain organic composites with high electrical conductance at low cost.

In this work we carried out a study of the electrical conductance of cw/PAni composites, at room temperature, prepared with different weights of PAni. It was observed that even small amounts of PAni cause a significant increase of the dc conductance. The present study is mainly concerned with this 'percolation' regime. The results show typical features of conduction in a disordered medium [7], usually displayed in a log-log plot of the complex conductivity as func- tion of the frequency: for the real component, a plateau followed by an almost linear rise, while the imaginary component steadily rises from the lowest frequency on. Superposed on this process, another one at lower frequencies was detected and disclosed in an Argand Impedance diagram, as a series process with the bulk one. It is attributed to the metal/composite interface. The results were analyzed by an equivalent conductance expression, which represents the bulk properties of the composite by the Random Free Energy Barrier model (RFEB model) [7-10], and the interface region between the polymer and the metallic electrode [11] by a simple equivalent circuit made by a capacitor in parallel with a resistor. The experimental-theoretical fittings provided the bulk and the interface contributions to the conductance showing that the bulk capacitance also increases as a function of the PAni concentration.

\section{Experimental procedure}

Doped Polyaniline was chemically obtained by a method describe elsewhere $[9,12]$, while $\mathrm{cw}$ was obtained by filtering carnauba wax from its suspension in water. Composites were prepared by mixing different weights of PAni and cw in a $x:(1-x)$ proportions $(0 \leq x \leq 1)$, and thus 
pressed under 10 tons. There resulted circular rigid pastilles of $5 \mathrm{~cm}^{2}, 1 \mathrm{~mm}$ thick, where circular aluminum electrodes $\left(\mathrm{A}=2.5 \mathrm{~cm}^{2}\right)$ were evaporated on both sides. Complex impedance measurements $\left(Z^{*}=Z \prime+i Z^{\prime \prime}\right)$ were carried out using a Solartron model SI 1260 in the $1 \mathrm{~Hz}$ to $1 \mathrm{MHz}$ frequency $(f)$ range at room temperature. Complex conductance $\left(\sigma^{*}=\sigma \prime+i \sigma^{\prime \prime}\right)$ was then obtained as $\sigma^{*}=1 /\left(Z^{*}\right)$.

\section{Experimental results}

Figs. 1.a, 1.b, 1.c and 1.d exhibit the real $(\sigma /)$ and imaginary $(\sigma ")$ components of the complex conductance for PAni concentration $(x)$, respectively equal to $0.0,0.10,0.50$ and 0.75 , as a function of the frequency $(f)$ in a log-log plot. While the imaginary component ever grows with the frequency, the real component shows a low frequency plateau, and for higher frequencies, the behavior $\sigma \prime(f) \propto f^{n}$, with $n$ close to 1 is observed. Usually these two regions merge smoothly at a critical frequency $f_{o}$, but this is not clear for $x=0$ (Fig. 1.a), most probably due to the superimposed noise. $f_{o}$ is around $2 \mathrm{KHz}$ for $x=0.10$, increasing with $x$ and reaching a value around $2 \times 10^{6} \mathrm{~Hz}$ for $x=0.75$. The dc conductance behaves similarly and changes from around $10^{-10} \Omega^{-1}$ (when $x=0.0$ ) to $2 \times 10^{-4} \Omega^{-1}$ (when $x=0.75$ ). This is the characteristic behavior of conduction in disordered solid [7-10], and has been observed to occur in conducting polymer conducting polymers [9], organic-inorganic composites [13], ceramics [14], glasses [15], and semiconductors materials [16]. From Fig. 1, it is also important to remark that some wavering occurs in both components around $f_{o} \approx 10^{3}$ $\mathrm{Hz}$, for all cases shown, even for $x=0.0$. For $x \geq 0.75$ (not shown here), the conductance of the samples presents a metallic characteristic: $\sigma \prime(f)$ is independent of $f$, and much higher than $\sigma$ " $(f)$, for the whole frequency range.

The experimental results in Fig. 1 were analyzed in an Argand impedance diagram (or Cole-Cole plot), as shown in Figs.2.a, 2.b, 2.c and 2.d. Fig. 2.a $(x=0.0)$ shows something like a quarter circle, with radius $\left(z_{0}\right)$ of around $10^{10} \Omega$, defining an impedance processes most probably associated to the disordered bulk conduction. On the other hand, Fig. 2.b $(x=0.10)$, shows two intercepting semicircles with $z_{0} / s$ around $2.5 \times 10^{6} \Omega$ and $2 \times 10^{6} \Omega$, which define two impedance processes: one at higher frequencies (low impedance values) and other at lower one (higher impedance values). The one at the left refers to the disordered bulk conduction, and therefore the one at the right, corresponding to the low frequency region, is attributed to a series process with the bulk one, related to an electrode process [11]. For $x=0.50$, Fig.2.c, and for $x=0.75$, Fig.2.d, only one deformed semicircle appears, with a lower $z_{0}$. For $x=0.50$, $z_{0}$ is around $5 x 10^{4} \Omega$, and for $x=0,75, z_{0}$ is around $7 \times 10^{3} \Omega$

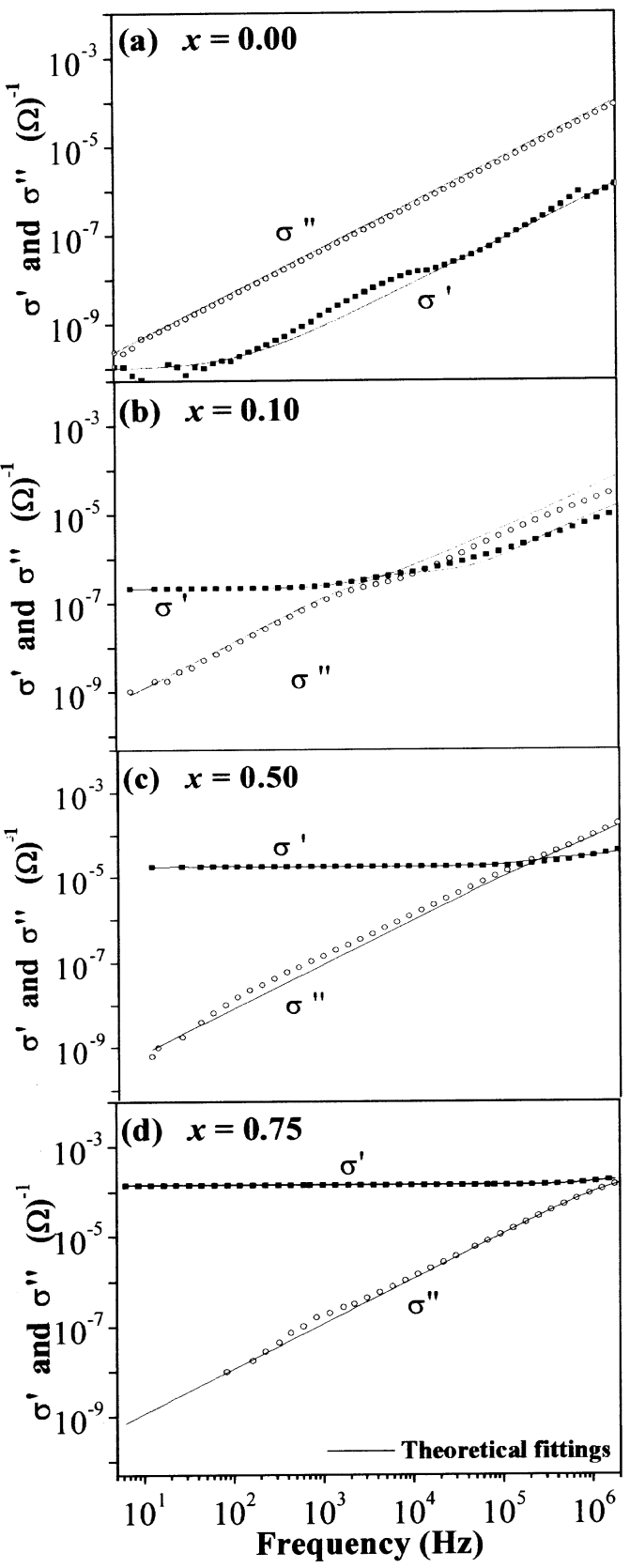

Figure 1. Real and imaginary components of the complex conductance (dots) and theoretical fitting (full lines) for $x$ equal to: (a) 0 . 0 , (b) 0.10 , (c) 0.50 and (d) 0.75 , at room temperature, where $x$ is the PAni proportion in the composite.

\section{Model of the ac bulk conductance}

J. C. Dyre has proposed the random free energy barrier model (RFEB) for grossly explaining conduction in disordered media [7,8]. According to this model the hopping carrier has to overcome thermically energy barriers randomly distributed between a minimum $W_{\min }$ and a maximum energy $W_{\max }$, uniformly dispersed through the sample bulk. For low frequencies the thermalized particle has enough time to meet high-energy barriers and the lowest 
jumping frequency $\gamma_{\min }$ dominates the process, with $\gamma_{\min }=$ $\gamma_{o} \exp \left(-W_{\max } / k T\right)$ where $\gamma_{o}$ is the escape frequency, $k$ the Boltzmann constant and $T$ the absolute temperature. This gives rise to the low frequency plateau region. For an electronic hopping process $\gamma_{o}$ depends exponentially on the hopping distance between the sites through the overlap integral of the localized wave functions. From the mean jumping frequency, the complex conductivity $\sigma^{*}(\omega)$ is obtained through a Fourier transform [9], where $\omega$ is the angular frequency $(2 \pi f)$. The following expression is then derived for the complex conductivity as a function of $\omega[7,8]$.

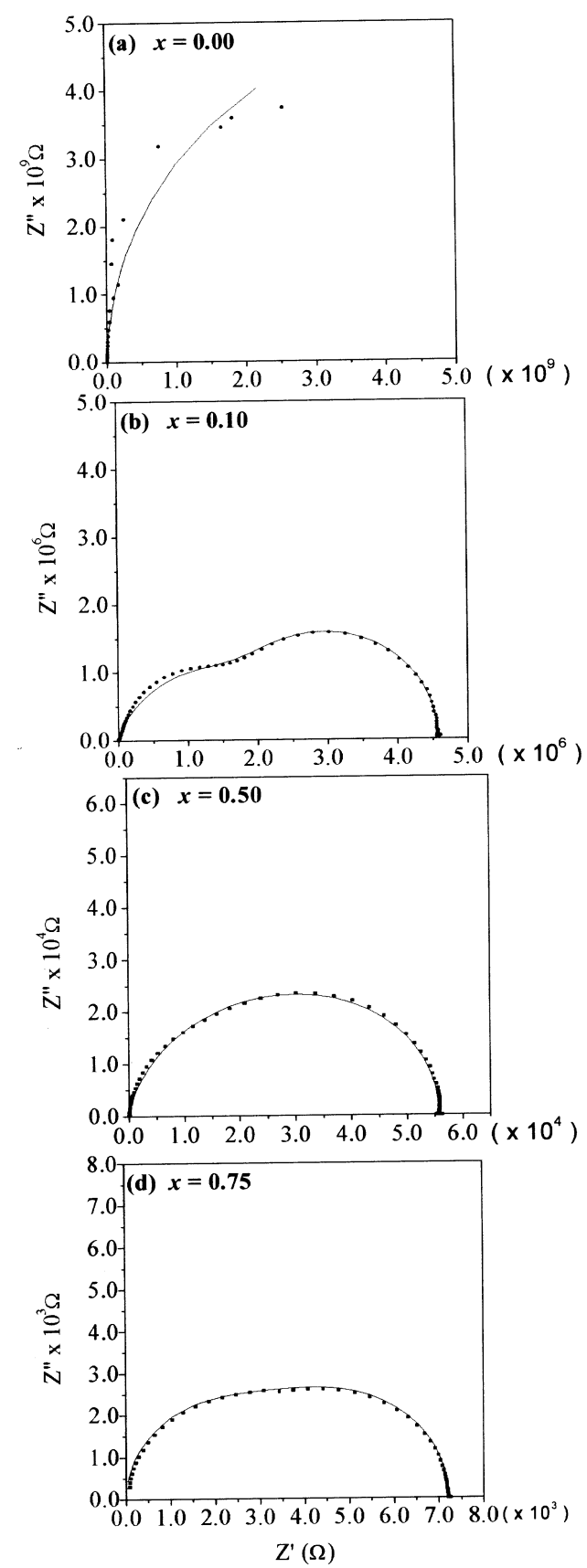

Figure 2. Argand diagram and theoretical fitting for $x$ equal to: (a) 0.0 , (b) 0.10 , (c) 0.50 and (d) 0.75 , at room temperature. Full lines represent the theoretical fittings.

$$
\sigma^{*}(\omega)=K\left[-i \omega+\frac{i \omega \ln \lambda}{\ln \left(\frac{1+i \omega / \gamma_{\min }}{1+i \omega / \gamma_{\max }}\right)}\right]
$$

where $\gamma_{\max }=\gamma_{0} \exp \left(-W_{\min } / K T\right), \lambda=\gamma_{\max } / \gamma_{\min }$ and $K$ takes account of the hopping nature of the process [17], depending on $\mathrm{T}$, on the hopping distance (a), on the density of carriers/atom $(c, 0<c<1)$, and on the electronic charge (e). $K$ is of the order of

$$
K \approx \frac{c e^{2}}{a k T}
$$

As already remarked, $\gamma_{0}$ - the escape frequency for an electronic hopping process - depends exponentially on the hopping distance and therefore we expect $\gamma_{0}$ to go as

$$
\gamma_{0} \approx \Omega_{0} e^{-\alpha a}
$$

with $\Omega_{0}$ and $\alpha$ constants. Returning to Eq. 1, its real part leads to a second high frequency plateau, but if we assume with Dyre [9] that $\gamma_{\max }>>\gamma_{\min }$, only the one at low frequency will be present, and Eq. 1 is simplified to [7,9]

$$
\sigma^{*}(\omega)=\sigma(0)\left[\frac{i \omega / \gamma_{\min }}{\ln \left(1+i \omega / \gamma_{\min }\right)}\right]
$$

This is the RFEB's equation [7,9], and $\sigma(0)$ is obtained taking the limit when $\omega \rightarrow 0$, i. e., the dc conductivity:

$$
\sigma(0)=K \gamma_{\min } \ln \lambda
$$

In this work, the concentration $x$ of PAni was significantly varied and the value of $\sigma(0)$ changed considerably. Eq. 3 suggests that this may be explained supposing that PAni introduces new hopping centers, decreasing the hopping distance. We then have $a \approx x^{-1 / 3}$, making $\gamma_{0}$ in Eq. 3 to grow with $x$. Still concerned with the dependence of $\sigma(0)$ on $x$, two cases may be asserted. First, it is possible that $\mathrm{c}$, the concentration of hopping carriers, also changes proportionally with $x$. In this case, using Eqs. 3-5 we would expect an overall dependence of $\sigma(0)$ on $x$ of the type

$$
\log \frac{\sigma(0)}{x^{4 / 3}} \approx x^{1 / 3}
$$

If instead the density of hopping carriers remains constant despite the increase of PAni dopping, we would have

$$
\log \frac{\sigma(0)}{x^{1 / 3}} \approx x^{1 / 3}
$$

Since the dependence of $\sigma(0)$ on $x$ in Eqs. 6 and 7 is of the exponential type, we do not expect the experimental results to be sufficiently accurate in order to allow a choice between the two cases.

Warning: in view of Eq. 2, $\sigma^{*}(\omega)$ in Eq. 4 is a conductivity. However, it will be treated hence forth as a conductance, which in the present case, differ from a conductivity by a constant geometric factor and therefore in a not essential way for the goals pursued here.

The total bulk conductance includes also the contribution from the dielectric polarization, which will be written 
simply as $i \omega C_{V}, C_{V}$ being the bulk capacitance and $i$ the imaginary unit. Therefore, the total bulk complex conductance $\sigma_{V}^{*}$ is obtained as a sum of conduction and of a dielectric contribution:

$$
\sigma_{V}^{*}(\omega)=\sigma^{*}(\omega)+i \omega C_{V}
$$

Finally, we note that the previously mentioned critical frequency $\omega_{o}\left(\omega_{o}=2 \pi f_{o}\right)$ is here $\gamma_{\min }$.

\section{The total conductance}

Let the conductance of the series electrode process, $\sigma_{S}^{*}$, be written as

$$
\sigma_{S}^{*}(\omega)=\sigma_{S R}+i \omega C_{S}
$$

where $\sigma_{S R}$ is the real conductance and $C_{S}$ the capacitance. Combining now in series the bulk conductance and the electrode conductance, Eqs. 8 and 9, the total conductance $\sigma^{*}(\omega)$ of the sample becomes:

$$
\sigma^{*}(\omega)=\frac{\sigma_{S}^{*}(\omega) \cdot \sigma_{V}^{*}(\omega)}{\sigma_{S}^{*}(\omega)+\sigma_{V}^{*}(\omega)}
$$

\section{Discussion}

The full curves in Fig. 1, for the ac conductance $\sigma^{*}(f)$, and in Fig. 2, for the impedances $z^{*}(f)=1 / \sigma^{*}(f)$, represent the best fitting achieved using Eq. 10. The parameters obtained are shown in Table I. It also includes the results for $x=0.15$ and 0.25 . For the fittings, the high frequency side of the real and the imaginary components of $\sigma^{*}(f)$ were first adjusted and from them the remaining parts were fixed. The reason for this was to see how good the RFEB expression, Eq. 4, describes the conduction process of such systems in the high frequency region. Additionally, this procedure helps to see if the representation of the electrode process as a resistor-capacitor circuit was sufficiently good to adjust the impedance and the conductance at low frequencies values. The agreement achieved is reasonable good, although some misfit is observed to occur in the high frequency side, showing that the RFEB model does not exactly explain the PAni/cw experimental results (perhaps the energy barriers are not uniformly distributed between $W_{\min }$ and $\left.W_{\max }\right)$. We note that the Argand plots were found to be very sensitive to changes in the parameter values. For higher doping ( $x \geq 0.50)$, the parameters in Table I were obtained almost solely from the Argand plot. It is also seen in Table I that $\sigma_{o}, \sigma_{S R}$ and $\gamma_{\min }$ seem to increase exponentially with $x$, as shown in Fig. 3, i.e. $\sigma_{0}$ is approximately proportional to $\gamma_{\min }$. From Eq. 5, this implies that $K \cdot \ln \lambda$ is nearly a constant, which is in agreement with ref. [9]. A roughly estimation from pure $\mathrm{cw}(x=0)$ indicates a value for the dielectric constant $\left(\varepsilon / \varepsilon_{0}\right)$ around $2.2[5,6]$, extracted from $C_{V}$ and the geometric factors (thickness $1 \mathrm{~mm}$ and area 2.5 $\mathrm{cm}^{2}$ ), as presented in Table I. For $x=0.75$, this physical parameter increases to 126 , a value relatively close to the pure doped polyaniline [9]. Concerning the surface conductance, which also increases exponentially with $x$, it probably derives from a chemical process involving chloride acid and the aluminum layer, which may introduces a dielectric junction between the composite layer and the aluminum electrode [18]. Finally, Table I shows that $C s$ is about a constant around $5 \times 10^{-10} \mathrm{~F}$. If we write for $C s, C s=k \varepsilon_{0} A / l$, there results for $l / k \sim 5 \times 10^{-4} \mathrm{~cm}$. If the dielectric constant of the interface is not much grater than $1, l$, the total interface thickness, will be of the order of $10^{-3} \mathrm{~cm}$ or $\sim 5 \mu \mathrm{m}$ each.

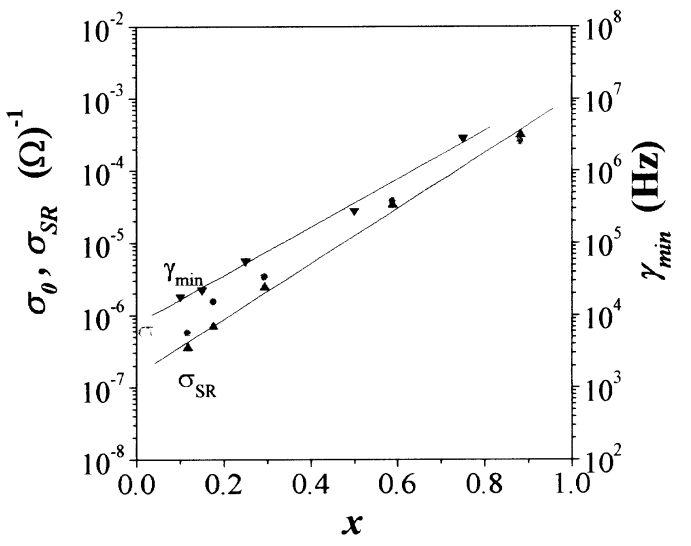

Figure 3. $\gamma_{\min }, \sigma_{o}$ and $\sigma_{S R}$ vs. $f$ obtained from Table I.

We now return to Eqs. 6 and 7. In principle, in order to compare the two possible behaviors expressed in these equations, we have first to make them non-dimensional, using as a reference one of the data. This was chosen as $x=0.10$ and therefore $\log \left[\left(\sigma_{0}(x) \cdot(0.10)^{n}\right) /\left(\sigma_{0}(0.10) \cdot x^{n}\right)\right]$ for $n=4 / 3$ and $n=1 / 3$ were plotted as a function of $x^{1 / 3}$, shown in Fig. 4, respectively in small circles and squares. As expected owing to the superimposed exponential dependence on $x$, the accuracy of our data is not sufficient to distinguish clearly between the two, since the resulting standard deviations, SD's, are about the same, 0.22 for $n=4 / 3$ and 0.20 for $n=1 / 3$. Any way we remind that the lower $n$ refers to a constant density of hopping carriers, independent of $x$.

\section{Conclusions}

In this paper we have measured the variation of the complex conductance of cw/PAni composites as a function of the amount of PAni. It was observed that even low amounts of PAni was able to increase the conductivity of the composite up to three orders of magnitude (see Table I), opening an alternative way for obtaining conducting organic composites at low cost. The results were interpreted using Dyre's RFEB model with moderate to good success. In serie with this bulk process, an electrode process was detected and modeled through a simple RC parallel combination. It was very interesting to see that even the undoped sample displays it, but it is unlike that such a process has a bearing on the decaying currents observed under a constant voltage [6]. 
Table I: Data resulting from fittings using Eq. 10 for samples with different $x$ proportions. $\varepsilon / \varepsilon_{o}$ is the bulk dielectric constant.

\begin{tabular}{ccccccc}
\hline$X$ & $\gamma_{\min } / 2 \pi(H z)$ & $\sigma_{o}(\Omega)^{-1}$ & $C_{V}(F)$ & $\sigma_{S R}(\Omega)^{-1}$ & $C_{S}(F)$ & $\varepsilon / \varepsilon_{o}$ \\
0.00 & 3.5 & $7.9 \times 10^{-9}$ & $5.0 \times 10^{-12}$ & $1.0 \times 10^{-10}$ & $2.4 \times 10^{-10}$ & 2.2 \\
0.10 & $1.8 \times 10^{3}$ & $6.0 \times 10^{-7}$ & $7.7 \times 10^{-12}$ & $3.4 \times 10^{-7}$ & $2.6 \times 10^{-10}$ & 3.5 \\
0.15 & $2.2 \times 10^{4}$ & $2.8 \times 10^{-6}$ & $8.0 \times 10^{-12}$ & $6.9 \times 10^{-7}$ & $1.1 \times 10^{-10}$ & 3.6 \\
0.25 & $5.6 \times 10^{4}$ & $7.5 \times 10^{-6}$ & $8.4 \times 10^{-12}$ & $2.4 \times 10^{-6}$ & $1.0 \times 10^{-10}$ & 3.8 \\
0.50 & $2.7 \times 10^{5}$ & $3.5 \times 10^{-5}$ & $3.1 \times 10^{-11}$ & $3.5 \times 10^{-5}$ & $2.1 \times 10^{-10}$ & 14.0 \\
0.75 & $2.8 \times 10^{6}$ & $4.0 \times 10^{-4}$ & $2.8 \times 10^{-10}$ & $2.1 \times 10^{-4}$ & $7.4 \times 10^{-10}$ & 126 \\
\hline
\end{tabular}

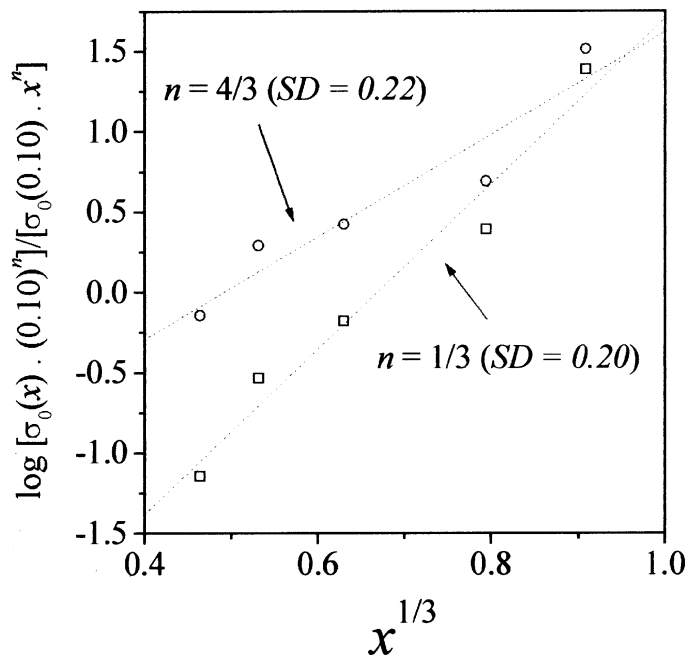

Figure 4. $\log \left[\left(\sigma_{0}(x) \cdot(0.10)^{n}\right) /\left(\sigma_{0}(0.10) \cdot x^{n}\right)\right]$ vs. $x^{1 / 3}$ for $n=$ $1 / 3$ or $4 / 3$ and the corresponding standard deviation (SD). Parameters from Table I.

\section{Acknowledgements}

This work was sponsored by FAPESP, CNPq, PROCAD/Capes and Millennium Science Initiative - MCT Brazil. On of us, G.F. Leal Ferreira thanks CNPq for a research grant.

\section{References}

[1] A.E. Job, C.J.L. Constantino, E.C. Camillo, M.Y. Teruya, N. Alves, L.H.C. Mattoso, Optimization of the electrical conductivity and the elastomeric performance for Polyaniline and natural rubber blends, submitted to Polymer (2002).
[2] S.J. Davies, T.G. Ryan, C.J. Wilde, G. Beyer, Synth. Metal 69, 209 (1995).

[3] C. Arbizzaani, M. Mastragostino, and B. Scrosati, in Handbook of Organic Conductive Molecules and Polymers, ed. by H.S. Nalwa, John Wiley \& Sons, Chap. 11 (1997).

[4] J.M. Ginder, A.F. Richter, A.G. MacDiarmid, A.J. Epstein, Sol. Stat. Comm. 63, 97 (1987).

[5] M. Eguchi, Proc. Phys. Math. Soc. Japan 1, 320 (1919).

[6] B. Gross, J. Chem. Phys. 17, 866 (1949).

[7] J.C. Dyre, T.B. Schroder, Rev. Mod. Phys. 72, 873 (2000).

[8] J.C. Dyre, Appl. Phys. 64, 2456 (1988).

[9] R.F. Bianchi, G.F. Leal Ferreira, C.M. Lepienski, and R.M. Faria, J. Chem. Phys. 110, 4602 (1999).

[10] G.F. Leal Ferreira and S.C. Costa, Braz. J. Phys. 29, 347 (1999).

[11] J. Ross Macdonald, J. Appl. Phys. 65, 4845 (1989).

[12] L.H.C. Mattoso, R.M. Faria, L.S. Bulhões, and A G MacDiarmid, Polym. Sci., Part A: Polym. Chem. 32, 2147 (1994).

[13] P.H. de Souza, R.F. Bianchi, K. Dahmouche, P. Judeinstein, R.M. Faria, T.J. Bonagamba, Chem. Mater. 13, 3685 (2001).

[14] R.F. Bianchi, J.A.G. Corrio, S.L. Cuffini, Y.P. Mascarenhas, R.M. Faria, Phys. Rev. 62, 10785 (2000).

[15] B. Roling, A. Happe, K. Funke, M.D. Ingram, Phys. Rev. Lett. 78, 2160 (1997).

[16] M. Pollak, T.H. Geballe, Phys. Rev. 122, 1742 (1961).

[17] A.J. Dekker, Solid State Theory, Prentice Hall Inc., Englewood, chap. 7 (1962).

[18] M. Fahlman, W.R. Salaneck, Surface Science 500, 904 (2002). 